\title{
Flexible Strip Supercapacitors for Future Energy Storage
}

\author{
Rui-Rong Zhang ${ }^{1} \quad$ Yan-Meng Xu$^{1} \quad$ David Harrison $^{1} \quad$ John Fyson $^{1} \quad$ Fu-Lian Qiu ${ }^{1}$ Darren Southee ${ }^{2}$ \\ ${ }^{1}$ College of Engineering, Design and Physical Sciences, Brunel University London, Uxbridge, UB83PH, UK \\ ${ }^{2}$ Loughborough Design School, Loughborough University, Leicestershire, UK
}

\begin{abstract}
Flexible strip supercapacitors are developed and their electrochemical properties are characterized. Activated carbon is used as the electrode material and it is found to have a good porous structure which provides a large surface area for energy storage. Furthermore, this activated carbon performs well. The manufacturing processes for the supercapacitors are described in detail and the preparation process has good reproducibility. The strip supercapacitors are combined in series and parallel to measure their electrical properties. The performances of these two samples in series or in parallel both follow the theoretical models. The electrochemical potential window of a series circuit of these two strip supercapacitors is $4.8 \mathrm{~V}$. The energy and power of the series or parallel circuits are equal to the sums of the two strip supercapacitors'.
\end{abstract}

Keywords: Strip supercapacitors, activated carbon, flexible, energy storage, characterisation.

\section{Introduction}

The high demand for improved efficiency and sustainability of energy systems in all aspects from generation up to end users is motivating the research and development of new storage systems and methods ${ }^{[1-8]}$. Supercapacitors (also named electrochemical capacitors or ultracapacitors), as energy storage devices with high power density, long cycle life and high reversibility, have been considered as a promising high power energy source for delivering peak power demands in portable electronic devices, electric vehicles and emergency power supplies. Supercapacitors can be generally classified into two types: 1) electrical double-layer capacitors (EDLCs) whose capacitances arise from charge separation at the electrode/electrolyte interface, such as carbon-based supercapacitors ${ }^{[3-7]}$, and 2) pseudocapacitors whose capacitances arise from reversible faradic reactions occurring at the electrode surface, such as transition metal oxide supercapacitors ${ }^{[3,8]}$. In EDLCs, the energy storage relies on the accumulation of charge at electrodes purely by electrostatics, and the charge is separated across the interface between the electrode and electrolyte. Thus, porous carbon materials, especially some activated carbons with a very high specific surface area and a suitable microtexture providing a high accessibility of electrolyte into the inner surface, are widely used for the electrode materials of $\operatorname{EDLCs}^{[4-7]}$.

Supercapacitors can deliver higher power than batteries and store more energy than conventional capacitors. Future developments are moving toward thinner, lighter

Regular paper

Special Issue on Recent Advance in Automation and Computing

Manuscript received December 30, 2013; accepted September 30 2014

This work was supported by European Union Seventh Framework Programme (FP7/2007-2013) (No. 81063).

Recommended by Associate Editor Yi Cao

(C) Institute of Automation, Chinese Academy of Science and Springer-Verlag Berlin Heidelberg 2015 and cheaper devices. However, many existing supercapacitors are still too bulky and heavy for their intended applications $^{[9,10]}$. It is a challenge to achieve highly efficient miniaturized supercapacitors, such as flexible supercapacitors, for good energy storage applications. Recently, there are reports of the flexible supercapacitors ${ }^{[9-15]}$, including fibre supercapacitors using pen ink as the active material ${ }^{[14]}$, flexible energy storage devices based on nano-composite paper ${ }^{[9]}$, fibre supercapacitors using $\mathrm{ZnO}$ nanowires as electrode ${ }^{[10]}$ and all-solid-state flexible microsupercapacitor on a chip ${ }^{[11]}$. In this work, flexible strip shaped supercapacitors are designed, manufactured and tested $^{[16]}$.

\section{Experimental}

\subsection{Preparation of EDLCs}

According to the published working mechanism ${ }^{[2]}$, the strip shaped supercapacitors are made by combining the two porous electrodes, the filter paper separator and the electrolyte solution, to make a strip supercapacitor as shown in Fig. 1.

\subsubsection{Electrode}

The electrodes consist of a stainless steel strip current collector (Fe/Cr18/Ni10/Mo3 with a thickness about $50 \mu \mathrm{m})$ coated with active materials. The active materials of the electrodes are prepared by mixing activated carbon (AC) with a binder (carboxymethyl cellulose, CMC). An activated carbon slurry is made as follows: A certain amount of CMC is added into water/ethanol (1:1) solvent at room temperature with magnetic stirring overnight to give a CMC concentration of $5 \% .0 .5 \mathrm{~g}$ of the activated carbon is added to the appropriate amount of CMC binder solution with magnetic stirring for 8 hours to obtain a homogeneous slurry with a $5 \%$ binder content (based on the total mass of solids). The stainless steel strip is cleaned with acetone. 
The thickness of the coated slurry is controlled by using a slot in plastic sheet of various thicknesses adhered to a surface with double side tape. Slurry is coated on the exposed surface of the stainless steel strip $(3 \mathrm{~mm} \times 100 \mathrm{~mm})$ using a sharp blade. The electrodes are dried after being coated with the slurry at room temperature overnight, then at $100{ }^{\circ} \mathrm{C}$ for 2 hours.

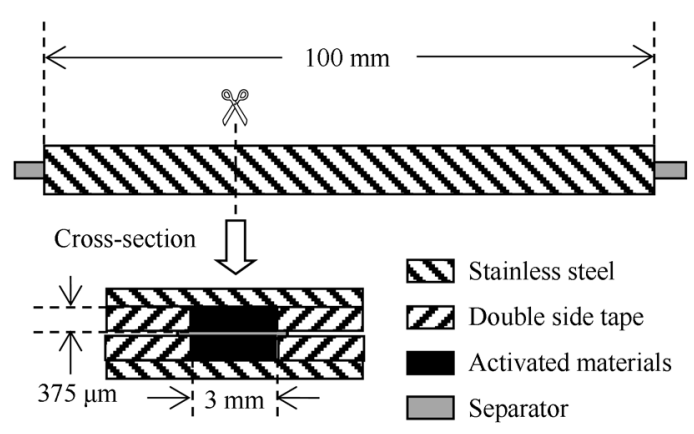

Fig. 1 The structure of a strip shaped EDLC

\subsubsection{Manufacture the strip shaped supercapaci- tors}

The electrolyte is made as follows. A $1 \mathrm{~mol} / \mathrm{L}$ solution of tetrabutylammonium tetrafluoroborate $\left(\mathrm{C}_{16} \mathrm{H}_{36} \mathrm{BF}_{4} \mathrm{~N}\right)$ in propylene carbonate $\left(\mathrm{C}_{4} \mathrm{H}_{6} \mathrm{O}_{3}\right)$ is made. The electrolyte is dropped on the surface of the coated activated materials, and these electrodes imbibed with electrolyte are kept under vacuum for 0.5 hour. Meanwhile, the filter paper to be used as a separator is wetted with the electrolyte.

\subsection{Determination of capacitance}

When a voltage is applied to an EDLC, opposite charges accumulate on the surfaces of the electrodes. The electrodes are separated by a separator to prevent short-circuits and thus an electric potential is produced between the two electrodes to achieve the function of energy storage. Capacitance $C$ is defined as the ratio of stored charge $Q$ to the applied voltage $V$ as

$$
C=\frac{\mathrm{d} Q}{\mathrm{~d} V}
$$

The performance of the strip shaped supercapacitors are measured by using standard cyclic voltammetry (CV) and galvanostatic charge-discharge methods, which are also widely used by other researchers ${ }^{[10,12,14-16]}$.

\subsubsection{Cyclic voltammetry}

A CV test is carried out by applying a positive (charging) voltage sweep $\mathrm{d} V / \mathrm{d} t$ (scan rate) in a specific voltage range and then reversing (discharging) the voltage sweep polarity immediately after the maximum voltage is achieved. As shown in Fig. 2 (a), the electrochemical behaviour of a strip shaped supercapacitor is evaluated based on the corresponding current response against the applied voltage. From the figure, the capacitance, $C 1$, can be calculated by

$$
C 1=\frac{Q_{\text {total }}}{2 \Delta V}
$$

where $Q_{\text {total }}$ is the supercapacitor's charge in coulombs, which is measured by the CV system used. $\Delta V$ is the voltage between the device's terminals in volts $(\mathrm{V})$.

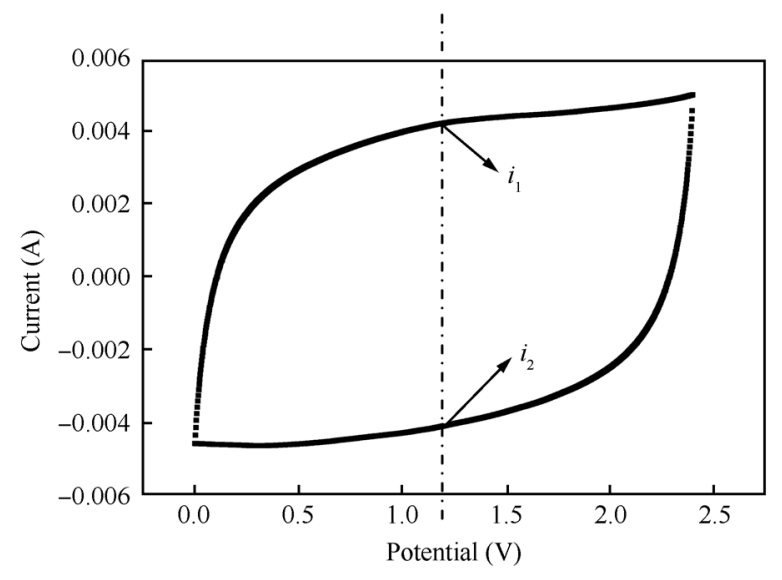

(a) Cyclic voltammogram of a strip shaped supercapacitor

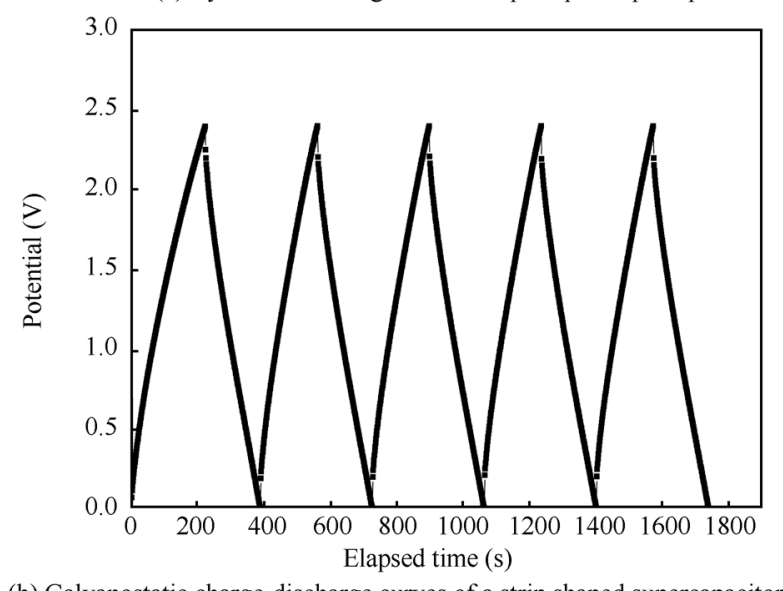

(b) Galvanostatic charge-discharge curves of a strip shaped supercapacitor

Fig. 2 Characterisation of a strip shaped supercapacitor

The capacitance, $C 2$, can be also calculated from the difference in the current at a point, usually the centre of the potential increasing and decreasing curves.

$$
C 2=\frac{\Delta i / 2}{\mathrm{~d} V / \mathrm{d} t}=\frac{\left|i_{1}-i_{2}\right| / 2}{\mathrm{~d} V / \mathrm{d} t}
$$

where $\Delta I$ is the total current $\left(\left|i_{1}-i_{2}\right|\right)$ of the CV curve and $\mathrm{d} V / \mathrm{d} t$ is the voltage scan rate $(\mathrm{V} / \mathrm{s})$.

\subsubsection{Galvanostatic charge-discharge}

A galvanostatic charge-discharge test is the most preferred DC test performed on supercapacitors for performance evaluation such as charge capacity and life span. The measurement consists of two steps: charging a supercapacitor at a constant current and discharging at a specific voltage range or charge-discharge time (see Fig. 2 (b)). The capacitance $C 3$ can be directly calculated by

$$
C 3=\frac{i \times \Delta t}{\Delta V}
$$

where $i$ is the discharge current in amperes (A) and $\Delta V$ is the voltage of the discharge $(\mathrm{V})$. 
$C 1, C 2$ and $C 3$ defined in (2) - (4) above are three different approaches to the measurement of the same capacitance. They are used to measure the capacitance of each sample.

Generally, the operating voltage is determined by the kind of electrolyte used in the supercapacitor ${ }^{[2,16,17]}$. The rated voltage includes a safety margin against the electrolyte's breakdown voltage at which the electrolyte decomposes or a chemical reaction will occur. Standard supercapacitors with aqueous electrolyte are normally specified with a rated voltage of $1.0 \mathrm{~V}$, and the working voltage of supercapacitors with organic solvents is about $2.5 \mathrm{~V}^{[2,16,17]}$. In this study, the electrolyte used is organic (1 mol/L solution of tetrabutylammonium tetrafluoroborate $\left(\mathrm{C}_{16} \mathrm{H}_{36} \mathrm{BF}_{4} \mathrm{~N}\right)$ in propylene carbonate $\left.\left(\mathrm{C}_{4} \mathrm{H}_{6} \mathrm{O}_{3}\right)\right)$. Therefore, the working voltage used for this kind of electrolyte is about $2.4 \mathrm{~V}$ under the safety margin.

\section{Results and discussion}

\subsection{Characterization of the activated car- bon}

Fig. 3 (a) shows the structure seen in scanning electron micrographs of the electrode activated materials. It can be seen that the size of the activated carbon particles ranges from hundreds of nanometers to tens of microns, with an average size of 20 microns. Fig. 3 (b) shows a magnified particle shown in Fig. 3 (a). A typical porous structure of AC materials can be observed. The diameter of holes on the porous particle is about $100 \mathrm{~nm}$. This microtexture of the AC material will allow good electrolyte accessibility to the inner surface of the carbon electrode. The porous structure also provides a large surface area for electrical charges storage and is beneficial for the specific capacitance value of EDLCs.

CV tests were conducted to characterize the electrochemical performance. All the cyclic voltammograms were recorded by a two-electrode system with a voltage window from 0 to $2.4 \mathrm{~V}$. Fig. 4 (a) reveals the CV curves of the supercapacitor at the scan rate from $5 \mathrm{mV} / \mathrm{s}$ to $40 \mathrm{mV} / \mathrm{s}$. The $\mathrm{CV}$ curves are close to a rectangle at the different scan rates of $5 \mathrm{mV} / \mathrm{s}, 10 \mathrm{mV} / \mathrm{s}$ and $20 \mathrm{mV} / \mathrm{s}$. This result illustrates a good electrochemical performance of the strip shaped supercapacitors. Different behaviours are found at the highest scan rate of $40 \mathrm{mV} / \mathrm{s}$. As shown in Fig. 4 (b), the capacitances calculated by (2) and (3) show the same trend: The capacitance decreases slightly with the increase of the scan rate. When the scan rates rise to $10 \mathrm{mV} / \mathrm{s}$ and $20 \mathrm{mV} / \mathrm{s}$, the capacitances are $0.2725 \mathrm{~F}$ and $0.2438 \mathrm{~F}$, respectively. They are almost equal to $80.7 \%$ and $72.2 \%$ of that measured at $5 \mathrm{mV} / \mathrm{s}$. Even at the scan rate of $40 \mathrm{mV} / \mathrm{s}$, the capacitance is still $0.1917 \mathrm{~F}$. The decrease of the capacitance at the higher scan rates implies a reasonable rate performance of the activated carbon.

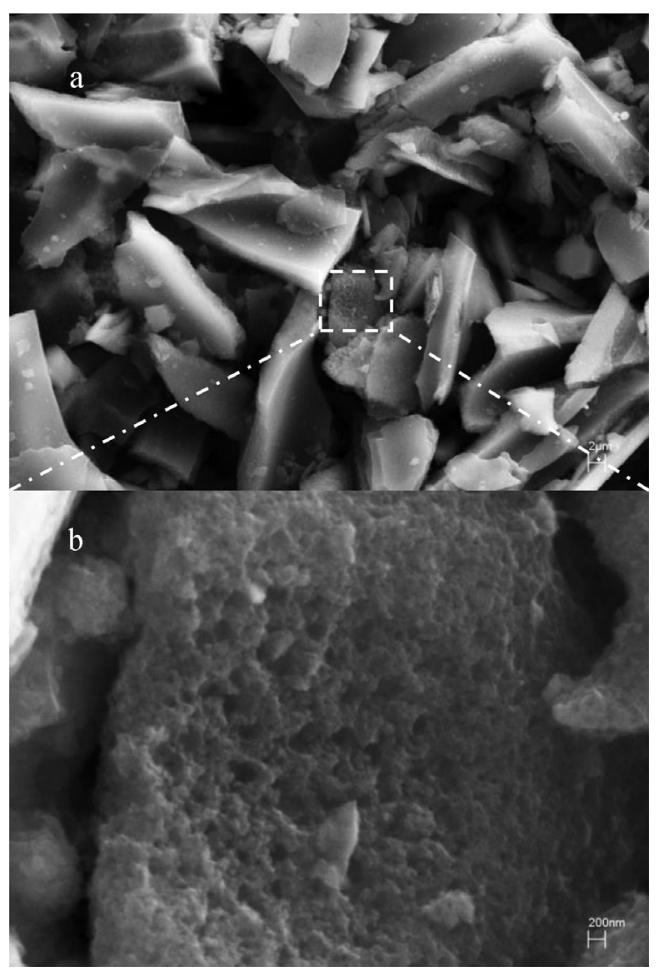

Fig. 3 The SEM images of activated materials layer

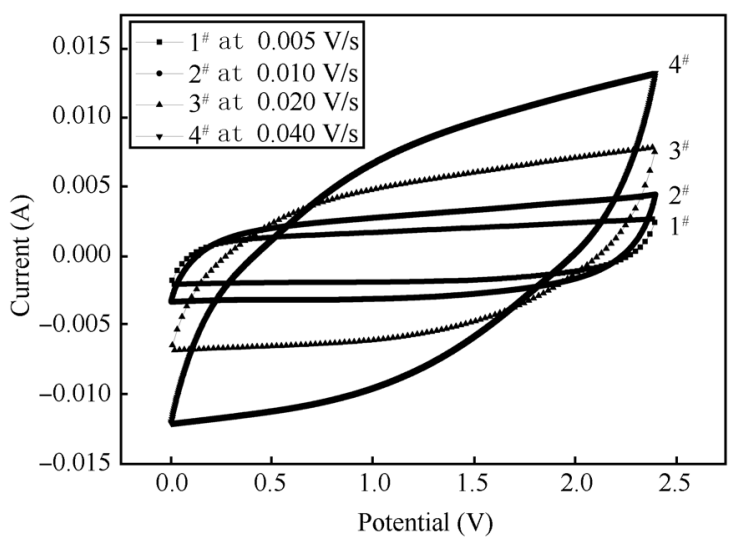

(a) Cyclic voltammogram of a sample at different scan rates

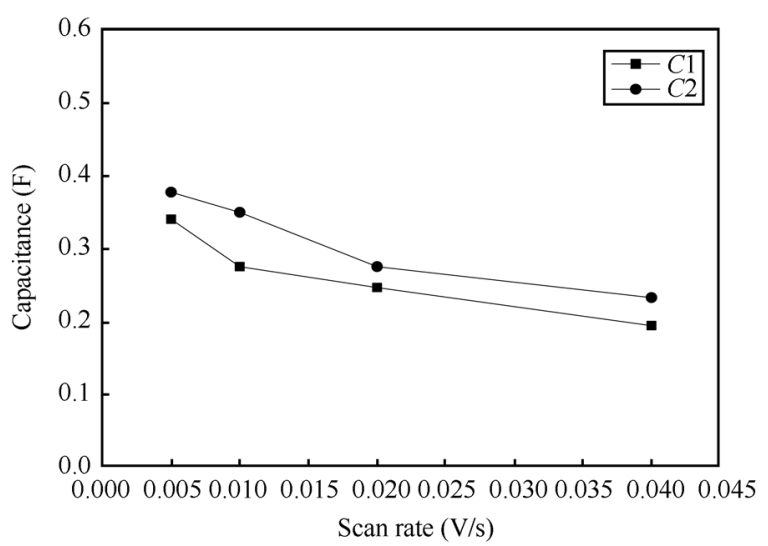

(b) Capacitances at different scan rates calculated by different equations

Fig. 4 Relationship between the capacitances calculated by different equations 


\subsection{Reproducibility of the manufacturing process}

In order to test if the manufacturing process for strip shaped EDLCs is reproducible or not, four EDLC samples were made under the same conditions. The size of the electrode is $3 \mathrm{~mm} \times 100 \mathrm{~mm}$ and the activated materials thickness is $375 \mu \mathrm{m}$. The binder content is $5 \%$ and the electrolyte concentration is $1.0 \mathrm{~mol} / \mathrm{L}$. Figs. 5 (a) and (b) show the characterization results by cyclic voltammetry. The four CV curves are ideal, which illustrates that all the strip shaped supercapacitors have a good electrochemical performance and there is little or no faradic reaction in the tested voltage range. The areas of these CV curves are similar (Fig. $5(\mathrm{a})$ ). Therefore, the capacitances calculated from the $\mathrm{CV}$ curves $(C 1$ and $C 2$ ) vary only slightly (Fig. $5(\mathrm{~b})$ ). The capacitances were also calculated from the charge/discharge curve $(C 3)$. Although the capacitance of each sample is measured by different approaches, $C 3$ shows the same trend as those calculated from the CV curves $(C 1$ and $C 2$ ). These results show that the manufacturing process for the strip shaped EDLCs described above has a good reproducibility.

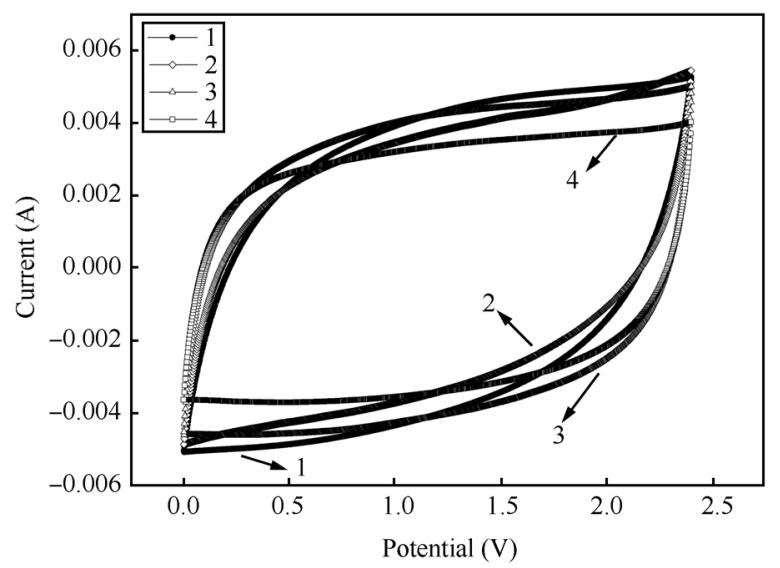

(a) Cyclic voltammograms of four samples with the same conditions

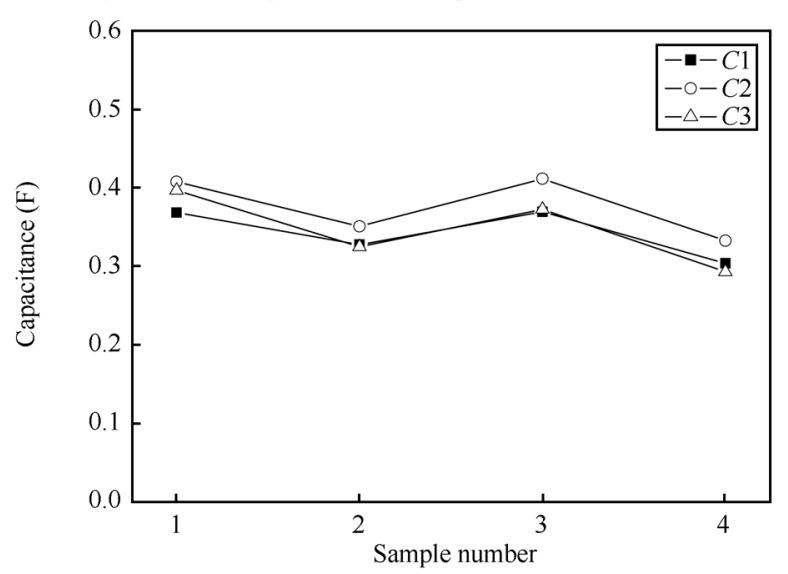

(b) Capacitances of the four samples calculated by different methods (CV curve and charge/discharge curve)

Fig. 5 Electrochemical performance characteristics of four samples with the same conditions
The average energy density and power density calculated for the four supercapacitors are about $8 \mathrm{Wh} / \mathrm{kg}$ and $500 \mathrm{~W} / \mathrm{kg}$, respectively. The normal energy density and power density of battery, electrostatic capacitor (normal capacitor) and supercapacitor are shown in Table $1^{[3-5]}$. The energy density of the strip shaped supercapacitor developed in this study is less than that of the battery (10$100 \mathrm{Wh} / \mathrm{kg}$ ) but much higher than that of the normal capacitor $(<0.1 \mathrm{Wh} / \mathrm{kg})$, and its performance is on the top end of supercapacitors ${ }^{[3]}$. The specific power density is more than that of battery $(50-200 \mathrm{~W} / \mathrm{kg})$, and less than that of normal capacitor $(>10000 \mathrm{~W} / \mathrm{kg})$.

Table 1 Comparison of the properties of battery, electrostatic capacitor, electrochemical capacitor and the strip supercapacitor developed in this work

\begin{tabular}{|c|c|c|c|c|}
\hline & Battery & $\begin{array}{c}\text { Electrostatic } \\
\text { capacitor }\end{array}$ & $\begin{array}{c}\text { Electrochemical } \\
\text { capacitor } \\
\text { (Supercapacitor) }\end{array}$ & $\begin{array}{c}\text { Strip } \\
\text { supercapacitor } \\
\text { developed in } \\
\text { this work }\end{array}$ \\
\hline $\begin{array}{l}\text { Specific } \\
\text { energy } \\
(\mathrm{Wh} / \mathrm{kg})\end{array}$ & $10-100$ & $<0.1$ & $1-10$ & 8 \\
\hline $\begin{array}{l}\text { Specific } \\
\text { power } \\
(\mathrm{W} / \mathrm{kg})\end{array}$ & $50-200$ & $>10000$ & $\approx 1000$ & 500 \\
\hline
\end{tabular}

\subsection{Combination of the strip shaped su- percapacitors}

Different combinations of supercapacitors in series or parallel are useful to obtain desired operating voltage and energy, so the supercapacitors can be used in a wide range of applications. To exploit the applicability and stability of the combinations of multiple strip supercapacitors, the performance of two strip supercapacitors and their electrical combinations in series and parallel were examined. Fig. 6 shows the CV curves of two single strip EDLCs (samples $a$ and $b$ ) and their series and parallel combination circuits. The working potential of the two strip EDLCs in parallel is the same as the working potential of the individual single samples, and half of that of samples in series which is extended to $4.8 \mathrm{~V}$. The area of CV curve of the parallel circuits is close to the total area of the two samples $a$ and $b$. The area of CV curve of the series circuits would be half of the total area of these two samples. The capacitances calculated by (2) from the CV curves are shown in Table 2. The capacitance of the parallel circuits is $0.395 \mathrm{~F}$, which is slightly less than the sum of the capacitances $(0.407 \mathrm{~F})$ of the two individual samples. The capacitance of series circuits is $0.094 \mathrm{~F}$, which is almost the same as the theoretically expected series capacitance of $0.098 \mathrm{~F}$. These results illustrate that the electrical properties of the series and parallel combinations of the EDLCs show a good agreement with the theoretical models of series and parallel circuit combinations. 


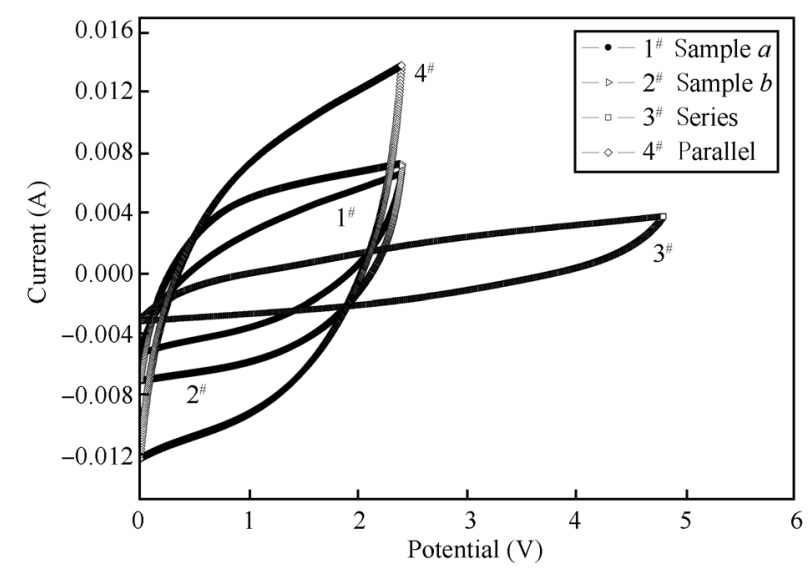

Fig. 6 Cyclic voltammograms recorded at $20 \mathrm{mV} / \mathrm{s}$ for two single strip supercapacitors and their electrical combinations in series and parallel

Table 2 Electrical series resistances (ESRs) from Nyquist plots and the capacitances from CV curve and galvanostatic charge/discharge curve with the ESRs and capacitances calculated using theory for series and parallel circuits

\begin{tabular}{ccccccc}
\hline \multicolumn{5}{c}{$\begin{array}{c}\text { Capacitance (F) } \\
(\mathrm{CV})\end{array}$} & $\begin{array}{c}\text { Capacitance }(\mathrm{F}) \\
(\mathrm{GCD})\end{array}$ & $\operatorname{ESR}(\Omega)$ \\
\multicolumn{5}{c}{$\begin{array}{c}\text { Experiment Theory } \\
\text { Experiment Theory Experiment Theory }\end{array}$} \\
\hline Sample $a$ & 0.165 & & 0.194 & & 9.3 & \\
Sample $b$ & 0.242 & & 0.301 & & 6.3 & \\
Series & 0.094 & 0.098 & 0.136 & 0.118 & 15.8 & 15.6 \\
Parallel & 0.395 & 0.407 & 0.493 & 0.495 & 4.3 & 3.8 \\
\hline
\end{tabular}

Fig. 7 shows the galvanostatic charge-discharge curves at charge currents of $5 \mathrm{~mA}$ for the two individual supercapacitors and the series circuit and of $10 \mathrm{~mA}$ for the parallel circuit. The potential window of the parallel circuit is $2.4 \mathrm{~V}$, the same as those of two single strip supercapacitors, and half of the series circuit. As shown in Table 2, the capacitances of the different cases calculated by (4) from the galvanostatic charge-discharge (GCD) curve show the same trend as those calculated from CV curves. Nyquist plots of the two single samples and the combinations in series or in parallel are shown in Fig. 8. It can be seen that the shapes of electrochemical impedance curves for the combination circuits are similar to those of the single strip EDLCs. It is observed that there is a sharp increase in the imaginary part of the impedance at the lower frequencies. This trend is consistent with a capacitive behaviour of an electrode. Meanwhile, the semicircular loops shown at high frequencies are probably associated with the porous structure of the AC materials ${ }^{[18]}$. The high intercept on the real axis is the electrical series resistance (ESR), as shown in Table 2. The capacitance values obtained from the experiments and theoretical calculations for the combinational models of series or parallel resistive and capacitive circuits are also given in Table 2. The capacitance of sample $b$ is $0.242 \mathrm{~F}$, which is about 1.47 times the sample $a^{\prime}$ s capacitance. It is easily found that the ESR of sample $a$ is also about 1.48 times that in sample $b$. For the case of the series circuit, the ESR is $15.8 \Omega$, which is very close to the sum of the ESRs of the two samples $a$ and $b$. The ESR of the parallel circuit is about $4.3 \Omega$, which is slightly higher than the total theoretical ESR of the parallel circuits of samples $a$ and $b$. These results are close to the expected ones for the two different circuits.

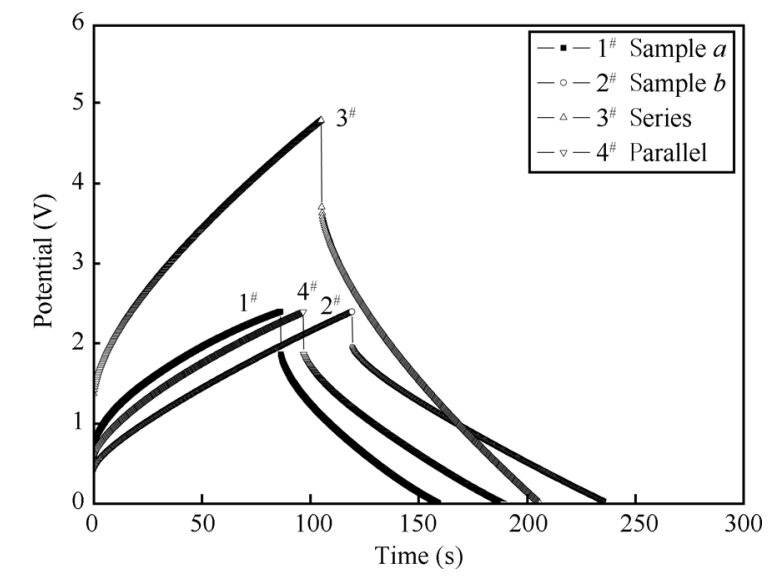

Fig. 7 Galvanostatic charge-discharge curves of the 3rd cycle of each case (for single and series circuit, $5 \mathrm{~mA}$ is used; for the parallel circuit, $10 \mathrm{~mA}$ is used)

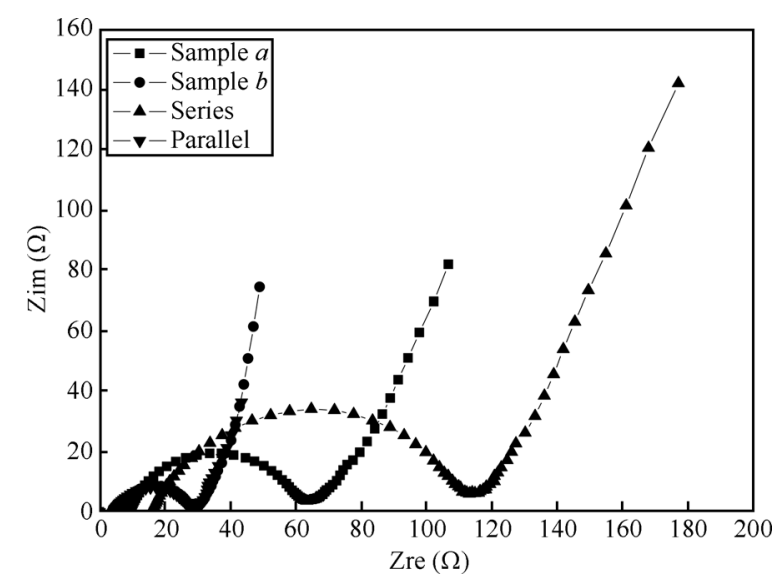

Fig. 8 Electrochemical performances of two single strip supercapacitors and their electrical combinations in series and parallel using a $4 \mathrm{mV}$ AC modulation for a frequency range of $100 \mathrm{kHz}$ to $0.01 \mathrm{~Hz}$

The energy $(E)$ and power $(P)$ of strip supercapacitors can be calculated by ${ }^{[14]}$

$$
\begin{aligned}
& E=\frac{1}{2} C V^{2} \\
& P=\frac{E}{t_{\text {discharge }}}
\end{aligned}
$$

where $V$ is the operating voltage and $C$ is the capacitance of the strip supercapacitors. The capacitance from the GCD curve together with the energy and power based on this capacitance are shown in Fig. 9. The energies of samples $a$ and 
$b$ are $0.559 \mathrm{~J}$ and $0.867 \mathrm{~J}$, respectively. The energy of the two strip EDLCs in parallel is $1.420 \mathrm{~J}$, which is very close to the sum of the energies of the two samples $(1.426 \mathrm{~J})$, while the energy of the two EDLCs in series has a slightly discrepancy $(1.567 \mathrm{~J})$ for this trend. Furthermore, the power of the two EDLCs in series is $0.0156 \mathrm{~W}$, very close to that of these samples in parallel $(0.0154 \mathrm{~W})$, which is very close to the sum of sample $a^{\prime}$ s $(0.0077 \mathrm{~W})$ and sample $b^{\prime}$ s power $(0.0074 \mathrm{~W})$.

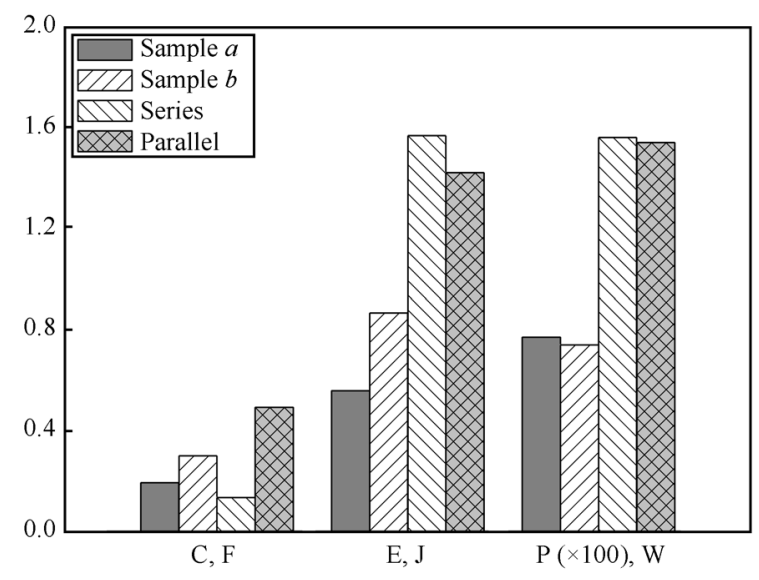

Fig. 9 Energy, power and capacitance of two single strip supercapacitors and their electrical combinations in series and parallel calculated from galvanostatic charge/discharge curve

\section{Conclusions}

The flexible strip shaped EDLCs $(3 \mathrm{~mm} \times 100 \mathrm{~mm})$ were successfully manufactured and characterized in this study. The AC material used is porous and has a lot of holes with an average size of $100 \mathrm{~nm}$, which allows good electrolyte accessibility to the inner surface of electrode and also provides a large surface area for charge storage. This kind of AC material also shows a good electrochemical scan rate performance. The manufacturing process has a good reproducibility. Two sample strip supercapacitors were combined in series and parallel to assess their electrical property as a power supplier. The performance of the series or parallel circuit showed a good agreement with the theoretical models for series and parallel circuit combinations. The strip supercapacitors can be used in various combinations of series or parallel to obtain desired energy or power for different applications. These narrow and thin strip supercapacitors enable the further development of highly flexible energy storage devices.

\section{References}

[1] M. K. Seo, S. Yang, I. J. Kim, S. J. Park. Preparation and electrochemical characteristics of mesoporous carbon spheres for supercapacitors. Materials Research Bulletin, vol. 45, no. 1, pp. 10-14, 2010.

[2] R. Kötz, M. Carlen. Principles and applications of electrochemical capacitors. Electrochimica Acta, vol. 45, no. 15-
16, pp. 2483-2498, 2000.

[3] Y. Zhang, H. Feng, X. B. Wu, L. Z. Wang, A. Q. Zhang, T. C. Xia, H. C. Dong, X. F. Li, L. S, Zhang. Progress of electrochemical capacitor electrode materials: A review. International Journal of Hydrogen Energy, vol. 34, no. 11, pp. 4889-4899, 2009.

[4] J. Gamby, P. L. Taberna, P. Simon, J. F. Fauvarque, M. Chesneau. Studies and characterisations of various activated carbons used for carbon/carbon supercapacitors. Journal of Power Sources, vol. 101, no. 1, pp. 109-116, 2001.

[5] D. Y. Qu. Studies of the activated carbons used in doublelayer supercapacitors. Journal of Power Sources, vol. 109, no. 2 , pp. 403-411, 2002.

[6] H. J. Shen, E. H. Liu, X. X. Xiang, Z. Z. Huang, Y. Y. Tian, Y. H. Wu, Z. L. Wu, H. Xie. A novel activated carbon for supercapacitors. Materials Research Bulletin, vol. 47, no. 3, pp. 662-666, 2012.

[7] L. M. Li, E. H. Liu, J. Li, Y. J. Yang, H. J. Shen, Z. Z. Huang, X. X. Xiang, L. Wen. A doped activated carbon prepared from polyaniline for high performance supercapacitors. Journal of Power Sources, vol. 195, no. 5, pp. 1516$1521,2010$.

[8] H. Gómez, M. K. Ram, F. Alvi, P. Villalba, E. Stefanakos, A. Kumar. Graphene-conducting polymer nanocomposite as novel electrode for supercapacitors. Journal of Power Sources, vol. 196, no. 8, pp. 4102-4108, 2011.

[9] V. L . Pushparaj, M. M. Shaijumon, A. Kumar, S. Murugesan, L. Ci, R. Vajtai, R. J. Linhardt, O. Nalamasu, P. M. Ajayan. Flexible energy storage devices based on nanocomposite paper. Proceedings of the National Academy of Sciences of the United States of America, vol.104, no. 34, pp. 13574-13577, 2007.

[10] J. Bae, M. K. song, Y. J. Park, J. M. Kim, M. Liu, Z. L. Wang. Fiber supercapacitors made of nanowirefiber hybrid structures for wearable/flexible energy storage. Angewandte Chemie International Edition, vol. 50, no.7, pp. 1683-1687, 2011.

[11] K. Wang, W. J. Zou, B. G. Quan, A. F. Yu, H. P. Wu, P. Jiang, Z. X. Wei. An all-solid-state flexible microsupercapacitor on a chip. Advanced Energy Materials, vol. 1, no. 6, pp. 1068-1072, 2011.

[12] G. Milczarek, A. Ciszewski, I. Stepniak. Oxygen-doped activated carbon fiber cloth as electrode material for electrochemical capacitor. Journal of Power Sources, vol. 196, no. 18 , pp. $7882-7885,2011$.

[13] X. Y. Zhang, X. Y. Wang, L. L. Jiang, H. Wu, C. Wu, J. C. $\mathrm{Su}$. Effect of aqueous electrolytes on the electrochemical behaviors of supercapacitors based on hierarchically. Journal of Power Sources, vol. 216, pp. 290-296, 2012.

[14] Y. P. Fu, X. Cai, H. W. Wu, Z. B. Lv, S. C. Hou, M. Peng, X. Yu, D. C. Zou. Fiber supercapacitors utilizing pen ink for flexible/wearable energy storage. Advanced Materials, vol. 24 , no. 42 , pp. 5713-5718, 2012. 
[15] X. Xiao, X. Peng, H. Y. Jin, T. Q. Li, C. C. Zhang, B. Gao, B. Hu, K. F. Huo, J. Zhou. Freestanding mesoporous VN/CNT hybrid electrodes for flexible all-solid-state supercapacitors. Advanced Materials, vol. 25, no. 36, pp. 50915097,2013

[16] R. R. Zhang, Y. M. Xu, D. Harrison, J. Fyson, F. L. Qiu, D. Southee. A study of flexible supercapacitors for future energy storage. In Proceedings of the 19th International Conference on Automation and Computing: Future Energy and Automation, IEEE, London, UK, pp. 87-90, 2013.

[17] A. Lewandowski, A. Olejniczak, M. Galinski, I. Stepniak. Performance of carbon-carbon supercapacitors based on organic, aqueous and ionic liquid electrolytes. Journal of Power Sources, vol. 195, no. 17, pp. 5814-5819, 2010.

[18] B. Yi, X. H. Chen, K. M. Guo, L. S. Xu, C. S. Chen, H. M. Yan, J. H. Chen. High-performance carbon nanotubeimplanted mesoporous carbon spheres for supercapacitors with low series resistance. Materials Research Bulletin, vol. 46, no. 11, pp. 2168-2172, 2011.

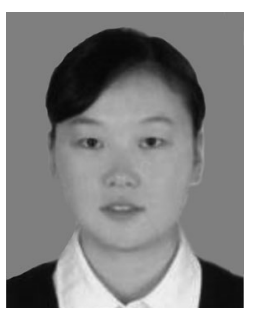

Rui-Rong Zhang received the B. Sc. degree in chemical engineering, and the M. Sc. degree in organic chemistry from Central South University, China in 2008 and 2011, respectively. She is currently a $\mathrm{Ph}$. D. candidate in design at the Brunel University London, UK.

Her research interests include electroals. chemistry and new energy storage materiE-mail: ruirong.zhang@brunel.ac.uk ORCID iD: 0000-0003-3200-8296

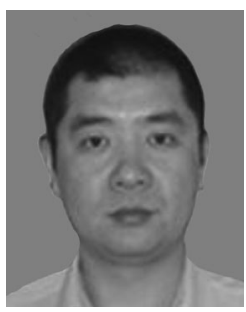

Yan-Meng $\mathrm{Xu}$ received the $\mathrm{Ph} . \mathrm{D}$. degree from the University of Southampton, UK in 2004. He is currently a lecturer at Brunel University London, UK.

His research interests include failure analysis for the engineering products, and printing materials and devices using the inkjet printing technology.

E-mail: yanmeng.xu@brunel.ac.uk (Cor-
ORCID iD: 0000-0001-5549-1079

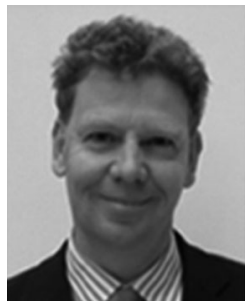

David Harrison received the B. Sc. degree in engineering science from Exeter University, UK in 1980 , and the $\mathrm{Ph}$. D. degree in robotics from Department of Electrical Engineering, Portsmouth Polytechnic, UK in 1988. He is now a professor at Brunel University London, UK.

His research interests in sustainable design and printed electronics.

E-mail: david.harrison@brunel.ac.uk

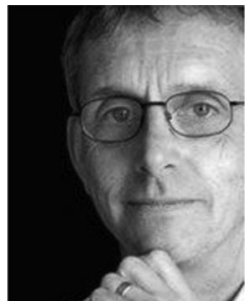

John Fyson received the B.Sc. degree in chemistry from Manchester. Kodak Research offered to pay him to do his hobby, photography, and therefore opted not to do a Ph. D. There, he stayed for the next 35 years. He is currently a visiting professor at Brunel University London, UK.

His research interests include design and testing of processing systems, optimizing chemistry and equipment and modelling complex chemical processes especially involving diffusion in thin substrates.

E-mail: john.fyson@brunel.ac.uk

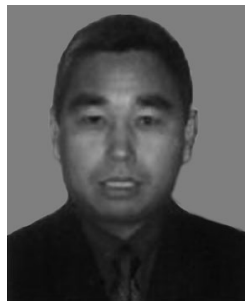

Fu-Lian Qiu is currently a research fellow at Brunel University London, UK.

His research interests include development of textiles for electrical energy generation and storage development of textiles for electrical energy generation and storage.

E-mail: fulian.qiu@brunel.ac.uk

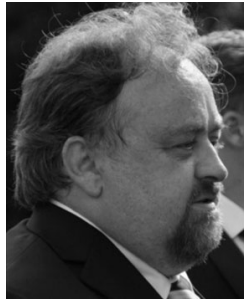

Darren Southee is currently a senior lecturer in design at Loughborough University, UK. His 21 year academic career has included roles for Brunel University London, UK, Bournemouth University, Bradford University (United Arab Emirates), UK, and the University of Lincoln, UK.

His research interests include the development of printed batteries, low power indicator displays, and energy storage in flexible systems.

E-mail: d.j.southee@lboro.ac.uk 